EXTRAPELVIC RECURRENCE RISK IN WOMEN WHO UNDERWENT MINIMALLY-INVASIVE VERSUS OPEN LAPAROTOMY FOR INTERMEDIATE-RISK ENDOMETRIAL CANCER; A MULTI-CENTER REVIEW

${ }^{1}$ A Dedinca*, ${ }^{2} \mathrm{~J}$ Song, ${ }^{3} \mathrm{H}$ Eckel, ${ }^{4} \mathrm{~T}$ Le, ${ }^{5} \mathrm{~L}$ Hopkins. ${ }^{1}$ University of Saskatchewan, Obstetrics and Gynecology, Saskatoon, Canada; ${ }^{2}$ University of Ottawa, Department of Radiation Oncology, Ottawa, Canada; ${ }^{3}$ University of Saskatchewan, College of Medicine, Saskatoon, Canada; ${ }^{4}$ University of Ottawa, Gynecologic Oncology, Ottawa, Canada; ${ }^{5}$ Saskatoon Cancer Centre, Gynecologic Oncology, Saskatoon, Canada

10.1136/ijgc-2021-IGCS.27

Objectives Objective: Minimally invasive surgery (MIS) is a common approach in endometrial cancer care. Recent studies have shown increased recurrence rates for women undergoing MIS versus laparotomy. Our goal was to assess endometrial cancer recurrence rates for MIS versus laparotomy.

Methods A multi-centre retrospective study was conducted for patients with intermediate-risk (ESGO) stage 1 endometrioid endometrial cancer treated surgically between January 1/2010December 31/2019. Surgical and pathology data were collected and oncology outcomes were assessed using Kaplan-Meier and multivariate COX regression analysis.

Results A total of 282 risk-stratified patients were reviewed from two major cancer centers. Median age of diagnosis was 64 and median follow up 60 months. A minimally invasive approach was completed for $65 \%$ of patients; $35 \%$ of patients underwent laparotomy. Adjuvant therapy with EBRT was completed in $21 \%$ of patients, brachytherapy in $48 \%$ and $31 \%$ had no adjuvant therapy. There was no difference in type of radiation between the two groups. In the MIS group $9.2 \%$ had any recurrence; $4.3 \%$ of these were extrapelvic $(p=0.154)$. In the laparotomy group $4.1 \%$ had any recurrence; $1 \%$ of these were extrapelvic $(p=0.169)$. Although no observed difference in overall survival, mean progression free survival was 106 months in the MIS group versus 117 months in the laparotomy group $(p=0.031)$. There was a significantly greater risk of extrapelvic relapse with MIS $(p=0.019)$.

Conclusions

Conclusion Among women with intermediate risk endometrial cancer, we observed a higher recurrence rate and risk of extrapelvic recurrence with MIS surgery. This finding is concerning and consistent with other published data.

\section{OP011/\#250 POSTMENOPAUSAL STATUS AND ELEVATED SERUM LEVEL OF CANCER ANTIGEN 125 SIGNIFICANTLY PREDICTED CONCURRENT ENDOMETRIAL CANCER IN WOMEN DIAGNOSED WITH ATYPICAL ENDOMETRIAL HYPERPLASIA BEFORE SURGERY}

Y-C Lou, J Guan*, X Chen. Obstetrics and Gynecology Hospital of Fudan University, Gynecology, Shanghai, China

10.1136/ijgc-2021-IGCS.28

Objectives About 10-66\% of patients with atypical endometrial hyperplasia diagnosed before surgery (pre-AEH) are found to have concurrent endometrial cancer (EC) after definitive hysterectomy, leading to incomplete primary surgery and delayed adjuvant treatment. This study aims to investigate which factors could predict underlying cancer risk in pre-AEH patients.

Methods All patients diagnosed with AEH by endometrial sampling and then underwent definitive hysterectomy from January 2016 to December 2019 were identified. Patients diagnosed with EC by final pathology were divided into 2 subgroups according to NCCN guideline 2021: low-risk, and intermediate-high risk.

Results Totally 624 pre-AEH patients were identified, 30.4\% $(n=190)$ of them were diagnosed with EC finally. Univariate analysis showed underlying risk of EC was correlated with postmenopausal status, higher CA125 serum level $(\geq 35 \mathrm{U} / \mathrm{ml})$, higher serum level of fast blood glucose $(\geq 7.0 \mathrm{mmol} / \mathrm{L})$ and older age ( $>50$ years old). In multivariate analysis, only postmenopausal status and CA125 $\geq 35 \mathrm{U} / \mathrm{ml}(\mathrm{OR}=3.89$, 95\% CI $=1.59-9.53 ;$ OR $=3.11,95 \%$ CI $=1.13-8.59)$ independently predicted concurrent EC. Remarkably, patients with postmenopausal status + CA125 $\geq 35 \mathrm{U} / \mathrm{ml}$ had significantly increased risk of finally-diagnosed EC $(\mathrm{OR}=14.10,95 \% \mathrm{CI}$ $=1.59-125.22$ ). Similar results were also found in predicting intermediate-high-risk EC. Notably, among all the postmenopasal patients, pre-AEH women with postmenopausal time $\geq$ 5 years were found to have highest risk of concurrent EC.

Conclusions Pre-AEH patients with postmenopausal status and elevated level of CA125 may have high risk of concurrent EC. More detailed evaluation before surgery should be suggested.

\section{OP012/\#257 MINIMALLY INVASIVE SURGERY IS ASSOCIATED WITH AN INCREASED RISK FOR LOCAL RECURRENCE IN HIGH-GRADE ENDOMETRIAL CARCINOMA}

${ }^{1} \mathrm{G}$ Levin*, ${ }^{2} \mathrm{~L}$ Kogan, ${ }^{3} \mathrm{~L}$ Helpman, ${ }^{4} \mathrm{R}$ Eitan, ${ }^{5} \mathrm{Z}$ Vaknin, ${ }^{6} \mathrm{O}$ Lavie, ${ }^{7} \mathrm{~A}$ Ben Arie, ${ }^{8} \mathrm{~A}$ Amit, ${ }^{9} \mathrm{~T}$ Levy, ${ }^{10} \mathrm{~A}$ Namazov, ${ }^{11}$ I Ben Shachar, ${ }^{12}$ | Atlas, ${ }^{13}$ | Bruchim, ${ }^{1} \mathrm{~T}$ Perri, ${ }^{14} \mathrm{O}$ Gemer. ${ }^{1}$ hadassah medical center, Gynecologic Oncology, Jerusalem, Israel; ${ }^{2}$ Hadassah, Gynecologic Oncology, Jerusalem, Israel; ${ }^{3}$ Cheba medical center, Gynecologic Oncology, Ramat Gan, Israel; ${ }^{4}$ Rabin Medical Center, Gynecology, Petah Tikva, Israel; ${ }^{5}$ Assaf Haroffe Medical Center, Sackler School of Medicine, Gynecology, Zrifin, Israel; ${ }^{6}$ Carmel Medical Center, Obstetrics and Gynecology, Haifa, Israel; ${ }^{7}$ 2. Kaplan Medical Center, Hebrew University, Gynecology, Rehovot, Israel; ${ }^{8}$ Rambam, Gymecologic Oncology, Haifa, Israel; ${ }^{9} 8$. Wolfson Medical Center, Holon, Sackler Faculty of Medicine, Tel Aviv University, Gynecology, Tel Aviv, Israel; ${ }^{10}$ Ahmet, Gynecology, Ashkelon, Israel; ${ }^{11}$ Zlv, Gynecologic Oncology, Zefat, Israel; ${ }^{12}$ Poriah, Gynecologic Oncology, Tiberia, Israel; ${ }^{13}$ Hillel Yafe, Gynecology, Hadera, Israel; ${ }^{14}$ Barzilai Medical Center, Gynecology, Ashkelon, Israel

\subsection{6/ijgc-2021-IGCS.29}

Objectives To compare oncological outcomes of women with high-grade endometrial carcinoma (HGEC) who underwent surgery by minimally invasive surgery (MIS) versus laparotomy.

Methods A retrospective cohort study performed in an academic multi-center setting. Consecutive women with HGEC cancer treated at 11 Israeli institutions between 2002 and 2017 were accrued in an assimilated database with a median follow-up of 52 months (range 12-120 months). Women with HGEC were stratified into two groups by route of surgery; MIS vs. laparotomy by an intention to treat. Clinical, pathological and outcome data were compared.

Results Six hundred and seventy-eight women met the inclusion criteria: 160 underwent MIS and 518 laparotomy. The two groups were comparable in demographic and clinical characteristics. Brachytherapy rate was similar in both groups 